\title{
Probabilistic representation for mild solution of the Navier-Stokes equations
}

\author{
C. Olivera* \\ Key words and phrases. Navier-Stokes equations, Stochastic differential \\ equation, Iyer-Cosntantin representation formula, Mild Solution.
}

\begin{abstract}
This paper is based on a formulation of the Navier-Stokes equations developed by Iyer and Constantin [6], where the velocity field of a viscous incompressible fluid is written as the expected value of a stochastic process. Our contribution is to establish this probabilistic representation formula for mild solutions of the Navier-Stokes equations on $\mathbb{R}^{d}$.
\end{abstract}

\section{Introduction}

We consider the classical Cauchy problem for the Navier-Stokes system, describing the evolution of a velocity field $u$ of an incompressible fluid with kinematic viscosity $\nu$

$$
\left\{\begin{array}{l}
\partial_{t} u(t, x)=\nu \Delta u(t, x)-[u(t, x) \cdot \nabla] u(t, x)-\nabla \pi(t, x) \\
\operatorname{div} u(t, x)=0 \\
u(0, x)=u_{0}(x)
\end{array}\right.
$$

The unknown quantities are the velocity $u(t, x)=\left(u_{1}(t, x), \ldots, u_{d}(t, x)\right)$ of the fluid element at time $t$ and position $x$ and the pressure $\pi(t, x)$. Such equations always attract the attention of many researchers, with an enormous quantity of publications in the literature. Concerning classical results about

*Departamento de Matemática, Universidade Estadual de Campinas, Brazil. E-mail: colivera@ime.unicamp.br. 
(1.1), we refer to the book by Teman [22]. In the 1960s, mild solutions were first constructed by Kato and Fujita ([13] and [14]) that are continuous in time and take values in the Sobolev spaces $u \in C\left([0, T], H^{s}\left(\mathbb{R}^{d}\right)\right)(s \geq$ $\left.\frac{d}{2}-1\right)$. Results on the existence of mild solutions with value in $L^{p}$, were established by Fabes, Jones and Riviere [12] and by Giga [15]. In 1992, a modern treatment for mild solutions in $H^{s}$ was given by Chemin [7]. For recent developments see Lemarie-Rieusset [19]. One of the (still open) million dollar problems posed by the Clay Institute is to show that given a smooth initial data $u_{0}$ the solution to (1.1) in three dimensions remains smooth for all time. We are interested in developing probabilistic techniques, that could help solve this problem

Probabilistic representations of solutions of partial differential equations as the expected value of functionals of stochastic processes date back to the work of Einstein, Feynman, Kac, and Kolmogorov in physics and mathematics. The Feynman-Kac formula is the most well-known example, which has provided a link between linear parabolic partial differential equations and probability theory, see [17. In 2008 Constantin and Iyer [6] (see also [5] and [16]) established a probabilistic Lagrangian representation formula by making use of stochastic flows. They show that $u$ is classical solution to the Navier-Stokes equation (1.1) if an only if $u$ satisfies the stochastic systems

$$
\begin{gathered}
X_{t}(x)=x+\int_{0}^{t} u\left(r, X_{r}(x)\right) d r+B_{t}, \\
u(t, x)=\mathbb{P E}\left[\left(\nabla X_{t}^{-1}\right)^{*} u_{0}\left(X_{t}^{-1}\right)\right]
\end{gathered}
$$

where $B_{t}$ denoting the standard Brownian motion, $\mathbb{P}$ is the Leray-Hodge projection and $*$ denotes the transposition of matrix. We mention that Fang, D Luo [9] obtained formula (1.3) on a compact manifolds, Rezakhanlou [21] wrote the representation (1.3) in the context of symplectic geometry and Zhang [25] extended the formula (1.3) for non-local operators. Different probabilistic representations of the solution of the the Navier-Stokes equations were studied by S. Albeverio, Y. Belopolskaya [1], Busnello [2], Busnello, Flandoli, Romito [3] Cipriano, Cruzeiro [4], Cruzeiro, Shamarova [8] and Zhang [25].

Strong solutions to the equation (1.2) are known for irregular $u$, the best result (after previous investigations of Zvonkin [26], Veretennikov[23], among 
others) being proved by Krylov, Röckner in [18]. More recently Flandoli, Gubinelli, Priola, see [10] and [11, proved that if the drift term is Hölder continuous then $x \rightarrow X_{s, t}$ is a $C^{1}$ - stochastic flow.

The contribution of this paper is to show that the unique mild solution of the equation (1.1) with values in $C\left([0, T], H^{s}\left(\mathbb{R}^{d}\right)\right)$ has the stochastic representation (1.3). The proof is simple and it is based in stability properties of the mild solution and in the flow properties associated to the equation (1.2). The result is the following theorem.

In fact, through of this paper, we fix a stochastic basis with a $d$-dimensional Brownian motion $\left(\Omega, \mathcal{F},\left\{\mathcal{F}_{t}: t \in[0, T]\right\}, \mathbb{P},\left(B_{t}\right)\right)$. We denoted $M$ a generic constant.

\section{Preliminaries}

\subsection{Mild Solution.}

In this subsection we recall some results on the Stokes operator.

$$
L_{\sigma}^{2}=\text { the closure in }\left[L^{2}\left(\mathbb{R}^{d}\right)\right]^{d} \text { of }\left\{u \in\left[C_{0}^{\infty}\left(\mathbb{R}^{d}\right)\right]^{d} \text {, divu }=0\right\}
$$

and

$$
G^{2}=\left\{\nabla q, q \in W^{1,2}(\mathbb{R})\right\}
$$

We then have the following Helmholtz decomposition

$$
\left[L^{2}(\mathbb{R})\right]^{d}=L_{\sigma}^{2} \oplus G^{2},
$$

the sum above reduces to the orthogonal decomposition and $L_{\sigma}^{2}$ is a separable Hilbert space, whose scalar product is denoted by $(\cdot, \cdot)$.

Let $\mathbb{P}$ be the continuous projection from $L^{2}\left(\mathbb{R}^{d}\right)$ to $L_{\sigma}^{2}$ associated with this decomposition and let $\Delta$ be the Laplace operator. Now, we define the Stokes operator $A$ in $L_{\sigma}^{2}$ by $A=-\mathbb{P} \Delta$. The operator $-A$ generates a bounded analytic semigroup $\{S(t)\}_{t \geq 0}$, see [20].

The potential space $H^{s}\left(\mathbb{R}^{d}\right)$ is defined as the space $(I-\Delta)^{-s / 2} L^{2}$ equipped with the norm $\|f\|_{H^{s}}:=\left\|(I-\Delta)^{s / 2}\right\|_{L^{2}}$. It is well know that 


$$
\|f g\|_{H^{s}} \leq M_{s}\|f\|_{H^{s}}\|g\|_{H^{s}} \quad \text { if } s>\frac{d}{2}
$$

We also recall that

$$
\begin{gathered}
\|S(t) u\|_{H^{s}} \leq M_{s}\|u\|_{H^{s}} \\
\left\|A^{\alpha} S(t) u\right\|_{H^{s}} \leq \frac{M_{s}}{t^{\alpha}}\|u\|_{H^{s}} .
\end{gathered}
$$

We consider the Navier -Stokes initial problem in the space $H^{s}\left(\mathbb{R}^{d}\right)$. Applying the projection operator $\mathbb{P}$ to (1.1) we get rid of the pressure term; setting $\nu=1$, equation (1.1) becomes

$$
\left\{\begin{array}{l}
d u(t)+A u(t) d t=B(u(t)) d t, \quad t>0 \\
u(0)=u_{0}
\end{array}\right.
$$

where the non linear term $B$ is defined by $B(u)=-\mathbb{P}[(u \cdot \nabla) u]$. Since $u$ is divergence free, we also have the representation $B(u)=-\mathbb{P}[\operatorname{div}(u \otimes u)]$ which will be useful later on. We consider the mild solution

Definition 2.1. A measurable function $u:[0, T] \rightarrow H^{s}\left(\mathbb{R}^{d}\right)$ is a mild solution of the equation (2.6) if

1. $u \in C\left(0, T ; H^{s}\left(\mathbb{R}^{d}\right)\right)$,

2. for all $t \in(0, T]$, we have

$$
u(t)=S(t) u_{0}+\int_{0}^{t} S(t-s) B(u(s)) d s
$$

Definition 2.2. We assume that there exist $T>0$ such that satisfies $u$ satisfies items 1 and 2 in definition 2.1. Then we called $u$ of local mild solution. 


\subsection{Stochastic Flows.}

In this subsection we follow the seminar paper by Flandoli, Gubinelli and Priola in [10]. We consider the SDE

$$
d X_{t}(x)=b\left(t, X_{s, t}(x)\right) d t+d B_{t}, X_{s}=x \in \mathbb{R}^{d},
$$

where $X_{s, t}(x)=X(s, t, x)$, also $X_{t}(x)=X(0, t, x)$. Moreover, the inverse $Y_{s, t}(x):=X_{s, t}^{-1}(x)$ satisfies the following backward stochastic differential equation

$$
Y_{s, t}(x)=x-\int_{s}^{t} b\left(r, Y_{r, t}(x)\right) d r-\left(B_{t}-B_{s}\right) .
$$

We denote by $\phi_{s, t}$ the flow associated to $X_{s, t}$ and $\psi_{s, t}$ its inverse. Let $T>0$ be be fixed. For any $\alpha \in(0,1)$, we denoted by $L^{\infty}\left([0, T], C_{b}^{\alpha}\left(\mathbb{R}^{d}\right)\right)$ the space bounded Borel functions $f:[0, T] \times \mathbb{R}^{d} \rightarrow \mathbb{R}$ such that

$$
\|f\|_{\alpha, T}:=\sup _{t \in[0, T]} \sup _{x \neq y,|x-y| \leq 1} \frac{|f(t, x)-f(t, y)|}{|x-y|^{\theta}}<\infty .
$$

We also recall the important results in [10].

Theorem 2.3. We assume that $b \in L^{\infty}\left([0, \infty), C_{b}^{\alpha}\left(\mathbb{R}^{d}\right)\right)$. Then

a) There exists a unique solution of the SDE (2.8).

b) There exists a stochastic flow $\phi_{s, t}$ of diffeomorphisms associated to equation (2.8). The flow is the class $C^{1+\alpha^{\prime}}$ for every $0<\alpha^{\prime}<\alpha$.

c) Let $b^{n} \in L^{\infty}\left([0, \infty), C_{b}^{\alpha}\left(\mathbb{R}^{d}\right)\right)$ be a sequence of the vector field and $\phi^{n}$ be the corresponding stochastic flow. If $b^{n} \rightarrow b$ in $L^{\infty}\left([0, \infty), C_{b}^{\alpha}\left(\mathbb{R}^{d}\right)\right)$, then for any $p \geq 1$ we have

$$
\begin{gathered}
\lim _{n \rightarrow \infty} \sup _{x \in \mathbb{R}^{d}} \sup _{s \in[0, T]} \mathbb{E}\left[\sup _{r \in[s, T]}\left|\phi_{s, r}^{n}-\phi_{s, r}\right|^{p}\right]=0, \\
\sup _{n} \sup _{x \in \mathbb{R}^{d}} \sup _{s \in[0, T]} \mathbb{E}\left[\sup _{r \in[s, T]}\left|D \phi_{s, r}^{n}\right|^{p}\right]<\infty, \\
\lim _{n \rightarrow \infty} \sup _{x \in \mathbb{R}^{d}} \sup _{s \in[0, T]} \mathbb{E}\left[\sup _{r \in[s, T]}\left|D \phi_{s, r}^{n}-D \phi_{s, t}\right|^{p}\right]=0 .
\end{gathered}
$$


Remark 2.4. The same results are valid for the backward flows $\psi_{s, t}^{n}$ and $\psi_{s, t}$ since are solutions of the same SDE driven by the drifts $-b_{n}$ and $-b$.

\section{Result}

Let $\left\{\rho_{n}\right\}_{n}$ be a family of standard symmetric mollifiers. We define the family of regularized initial data as $u_{0}^{n}(x)=\left(u * \rho_{\varepsilon}\right)(x)$. Let $T>0$. Now, we assume that for all $n$ there exist $u^{n}$ a classical solution in $[0, T] \times \mathbb{R}^{d}$ of

$$
\left\{\begin{array}{l}
d u^{n}(t)+A u^{n}(t) d t=B\left(u^{n}(t)\right) d t, \quad t>0 \\
u(0)=u_{0}^{n} .
\end{array}\right.
$$

Theorem 3.1. We fix $T>0$ and we assume $u_{0} \in H^{s}\left(\mathbb{R}^{d}\right)$. Let be $u \in$ $C\left([0, T], H^{s}\left(\mathbb{R}^{d}\right)\right)$ a local mild solution with $s>\frac{d}{2}$ such that $u^{n}$ converge to $u$ in $C\left([0, T], H^{s}\left(\mathbb{R}^{d}\right)\right)$. Then we have that

$$
u(t, x)=\mathbb{P} \mathbb{E}\left[\left(\nabla X_{t}^{-1}\right)^{*} u_{0}\left(X_{t}^{-1}\right)\right] .
$$

Proof. Step 1 : Regular initial data.

By Itô formula or by Constantin-Iyer [5] we have

$$
u^{n}(t, x)=\mathbb{P} \mathbb{E}\left[\left(\nabla Y_{t}^{n}\right)^{*} u_{0}^{n}\left(Y_{t}^{n}\right)\right] .
$$

where $Y_{t}^{n}$ is the inverse of

$$
d X_{t}^{n}=u^{n}\left(t, X_{t}^{n}\right) d t+d B_{t}, X_{0}=x \in \mathbb{R}^{d} .
$$

Step 2 : Convergence II.

From $H^{s}\left(\mathbb{R}^{d}\right) \subset C_{b}^{\alpha}\left(\mathbb{R}^{d}\right)$ with $\alpha=s-\frac{d}{2}$, hypothesis and theorem 2.3 we have

$$
\begin{gathered}
\lim _{n \rightarrow \infty} \sup _{x \in \mathbb{R}^{d}} \mathbb{E}\left[\sup _{t \in[0, T]}\left|Y_{t}^{n}-Y_{t}\right|^{p}\right]=0, \\
\lim _{n \rightarrow \infty} \sup _{x \in \mathbb{R}^{d}} \mathbb{E}\left[\sup _{t \in[0, T]}\left|D Y_{t}^{n}-D Y_{t}\right|^{p}\right]=0, \\
\quad \sup _{x \in \mathbb{R}^{d}} \mathbb{E}\left[\sup _{t \in[0, T]}\left|D Y_{t}^{n}\right|^{p}\right]<\infty,
\end{gathered}
$$


where $Y_{t}$ is the inverse of $X_{t}$ and it verifies (2.8) with drift $u(t, x)$.

Step 3 : Convergence III.

We observe that

$$
\begin{aligned}
& \mathbb{E}\left[\left(\nabla Y_{t}^{n}\right)^{*} u_{0}^{n}\left(Y_{t}^{n}\right)\right]-\mathbb{E}\left[\left(\nabla Y_{t}\right)^{*} u_{0}\left(Y_{t}\right)\right] \mid \\
& \leq \mathbb{E}\left[\left(\nabla Y_{t}^{n}\right)^{*} u_{0}^{n}\left(Y_{t}^{n}\right)-\left(\nabla Y_{t}^{n}\right)^{*} u_{0}\left(Y_{t}^{n}\right)\right] \mid \\
& +\mathbb{E}\left[\left(\nabla Y_{t}^{n}\right)^{*} u_{0}\left(Y_{t}^{n}\right)-\left(\nabla Y_{t}^{n}\right)^{*} u_{0}\left(Y_{t}\right)\right] \mid \\
& +\mathbb{E}\left[\left(\nabla Y_{t}^{n}\right)^{*} u_{0}\left(Y_{t}\right)-\left(\nabla Y_{t}\right)^{*} u_{0}\left(Y_{t}\right)\right] \mid \\
& =I_{1}+I_{2}+I_{3} .
\end{aligned}
$$

By Hölder inequality and (3.17) we have

$$
\begin{aligned}
& \int_{\mathbb{R}^{d}}\left|I_{1}\right|^{2} d x \\
& \leq \int_{\mathbb{R}^{d}} \mathbb{E}\left|\nabla Y_{t}^{n}\right|^{2} \mathbb{E}\left|u_{0}^{n}\left(Y_{t}^{n}\right)-u_{0}\left(Y_{t}^{n}\right)\right|^{2} d x \\
& \leq \sup _{x, t} \mathbb{E}\left|\nabla Y_{t}^{n}\right|^{2} \int_{\mathbb{R}^{d}} \mathbb{E}\left|u_{0}^{n}\left(Y_{t}^{n}\right)-u_{0}\left(Y_{t}^{n}\right)\right|^{2} d x \\
& =C \int_{\mathbb{R}^{d}} \mathbb{E}\left|u_{0}^{n}(x)-u_{0}(x)\right|^{2} d x
\end{aligned}
$$

it follows that $I_{1} \rightarrow 0$ in $C\left([0, T], L^{2}\left(\mathbb{R}^{d}\right)\right)$.

By Hölder inequality and (3.17) we obtain

$$
\begin{aligned}
& \int_{\mathbb{R}^{d}}\left|I_{2}\right|^{2} d x \\
& \leq \int_{\mathbb{R}^{d}} \mathbb{E}\left|\nabla Y_{t}^{n}\right|^{2} \mathbb{E}\left|u_{0}\left(Y_{t}^{n}\right)-u_{0}\left(Y_{t}\right)\right|^{2} d x \\
& \leq \sup _{x, t} \mathbb{E}\left|\nabla Y_{t}^{n}\right|^{2} \int_{\mathbb{R}^{d}} \mathbb{E}\left|u_{0}\left(Y_{t}^{n}\right)-u_{0}\left(Y_{t}\right)\right|^{2} d x,
\end{aligned}
$$

from (3.15) and dominated convergence we get that $I_{2} \rightarrow 0$ in $C\left([0, T], L^{2}\left(\mathbb{R}^{d}\right)\right)$. 
We observe that

$$
\begin{aligned}
& \int_{\mathbb{R}^{d}}\left|I_{3}\right|^{2} d x \\
& \leq \int_{\mathbb{R}^{d}} \mathbb{E}\left|\left(\nabla Y_{t}^{n}\right)^{*}-\left(\nabla Y_{t}\right)^{*}\right|^{2} \mathbb{E}\left|u_{0}\left(Y_{t}\right)\right|^{2} d x \\
& \leq C \sup _{x, t} \mathbb{E}\left|\left(\nabla Y_{t}^{n}\right)^{*}-\left(\nabla Y_{t}\right)^{*}\right|^{2} d x
\end{aligned}
$$

from $(3.16)$ we deduce that $I_{3} \rightarrow 0$ in $C\left([0, T], L^{2}\left(\mathbb{R}^{d}\right)\right)$.

Thus we conclude that $\mathbb{E}\left[\left(\nabla Y_{t}^{n}\right)^{*} u_{0}^{n}\left(Y_{t}^{n}\right)\right] \rightarrow \mathbb{E}\left[\left(\nabla Y_{t}\right)^{*} u_{0}\left(Y_{t}\right)\right]$ strong in $C\left([0, T], L^{2}\left(\mathbb{R}^{d}\right)\right)$. This implies that $u^{n}=\mathbb{P E}\left[\left(\nabla Y_{t}^{n}\right)^{*} u_{0}^{n}\left(Y_{t}^{n}\right)\right]$ converge to $\mathbb{P E}\left[\left(\nabla Y_{t}\right)^{*} u_{0}\left(Y_{t}\right)\right]$ in $C\left([0, T], L^{2}\left(\mathbb{R}^{d}\right)\right)$.

Step 4: Conclusion.

From step I and hypothesis we conclude that $u(t, x)=\mathbb{P E}\left[\left(\nabla Y_{t}\right)^{*} u_{0}\left(Y_{t}\right)\right]$.

We observed that by Kato construction of the mild solution we can take $T$ sufficiently small such that there exists an unique mild solution in $C\left(0, T, ; H^{s}\left(\mathbb{R}^{d}\right)\right)$ with initial conditions $u_{0}$ and $u_{0}^{n}$, see for instance [19].

Corollary 3.2. We assume $u_{0} \in H^{s}\left(\mathbb{R}^{d}\right)$ and $T$ is small enough. Let be $u \in C\left([0, T], H^{s}\left(\mathbb{R}^{d}\right)\right)$ the unique local mild solution with $s>\frac{d}{2}$. Then we have that

$$
u(t, x)=\mathbb{P} \mathbb{E}\left[\left(\nabla X_{t}^{-1}\right)^{*} u_{0}\left(X_{t}^{-1}\right)\right]
$$

Proof. Now, we consider $u^{n}$ the unique local mild solution of

$$
\left\{\begin{array}{l}
d u^{n}(t)+A u^{n}(t) d t=B\left(u^{n}(t)\right) d t, \quad t>0 \\
u(0)=u_{0}^{n} .
\end{array}\right.
$$

in $[0, T]$. We have

$$
u(t)-u^{n}(t)=u_{0}-u_{0}^{n}+\int_{0}^{t} S(t-s)\left(B(u(s))-B\left(u^{n}(s)\right)\right) d s .
$$


By classical estimations we obtain

$$
\begin{aligned}
& \left\|u(t)-u^{n}(t)\right\|_{H_{s}} \\
& \leq\left\|u_{0}-u_{0}^{n}\right\|_{H_{s}} \\
& +\int_{0}^{t}\left\|S(t-s)\left(B(u(s))-B\left(u^{n}(s)\right)\right)\right\|_{H_{s}} d s \\
& \leq\left\|u_{0}-u_{0}^{n}\right\|_{H_{s}} \\
& +\int_{0}^{t} \frac{M}{(t-s)^{\frac{1}{2}}}\left(\left\|u^{n}(s)\right\|_{H_{s}}+\|u(s)\|_{H_{s}}\right)\left\|u(s)-u^{n}(s)\right\|_{H_{s}} d s .
\end{aligned}
$$

It is well know that

$$
\|u(t)\|_{H^{s}} \leq M\left\|u_{0}\right\|_{H^{s}}
$$

and

$$
\left\|u^{n}(t)\right\|_{H^{s}} \leq M\left\|u_{0}^{n}\right\|_{H^{s}} \leq M\left\|u_{0}\right\|_{H^{s}}
$$

Thus we have

$$
\begin{aligned}
& \sup _{t \in[0, T]}\left\|u(t)-u^{n}(t)\right\|_{H_{s}} \\
& \leq\left\|u_{0}-u_{0}^{n}\right\|_{H_{s}} M T^{\frac{1}{2}}\left(\sup _{t \in[0, T]}\left\|u^{n}(t)\right\|_{H_{s}}+\sup _{t \in[0, T]}\|u(t)\|_{H_{s}}\right) \\
& \times \sup _{t \in[0, T]}\left\|u(s)-u^{n}(s)\right\|_{H_{s}} \\
& \leq\left\|u_{0}-u_{0}^{n}\right\|_{H_{s}}+M T^{\frac{1}{2}}\left\|u_{0}\right\|_{H_{s}} \sup _{t \in[0, T]}\left\|u(s)-u^{n}(s)\right\|_{H_{s}} .
\end{aligned}
$$

If $M T^{\frac{1}{2}}\left\|u_{0}\right\|_{H_{s}}<1$ we get

$$
\sup _{t \in[0, T]}\left\|u(t)-u^{n}(t)\right\|_{H_{s}} \leq C\left\|u_{0}-u_{0}^{n}\right\|_{H_{s}} .
$$

Then we deduce

$$
\sup _{t \in[0, T]}\left\|u(t)-u^{n}(t)\right\|_{H_{s}} \rightarrow 0 \text { as } n \rightarrow \infty .
$$

The representation (3.18) we follow from the theorem 3.1 , 


\section{Acknowledgements}

Christian Olivera is partially supported by FAPESP by the grants 2017/176700 and 2015/07278-0 . Also supported by CNPq by the grant 426747/2018-6.

\section{References}

[1] S. Albeverio, Y. Belopolskaya, Generalized solutions of the Cauchy problem for the Navier-Stokes system and diffusion processes, Cubo, $12,2,2010$.

[2] B. Busnello, A probabilistic approach to the two dimensional NavierStokes equations, Ann. Probab. 27, 1750-1780, 1999.

[3] B. Busnello, F. Flandoli and M. Romito, A probabilistic representation for the vorticity of a $3 D$ viscous fluid and for general systems of parabolic equations, Proc. Edinb. Math. Soc. 48, 295-336, 2005.

[4] F Cipriano, AB Cruzeiro, Navier-Stokes Equation and Diffusions on the Group of Homeomorphisms of the Torus, Communications in Mathematical Physics, 275, 255-269, 2007.

[5] P Constantin, G Iyer, A stochastic Lagrangian representation of the three-dimensional incompressible Navier-Stokes equations , Ann. Appl. Probab, 21, 1466-1492, 2011.

[6] P Constantin, G Iyer, A stochastic-Lagrangian approach to the NavierStokes equations in domains with boundary Communications on Pure and Applied, 61, 330-345, 2008.

[7] J. M. Chemin, Remarques sur lexistence globale pour le syst'eme de Navier-Stokes incompressible, SIAM J. Math. Anal., 23, 20-28, 1992.

[8] AB Cruzeiro, E Shamarova, Navier-Stokes equations and forwardbackward SDEs on the group of diffeomorphisms of a torus, Stochastic processes and their applications, 119, 4034-4060, 2009.

[9] S Fang, D Luo, Constantin and Iyer's Representation Formula for the Navier-Stokes Equations on Manifolds , Potential Analysis, 48, 181-206, 2018. 
[10] F. Flandoli, M. Gubinelli, E. Priola, Well-posedness of the transport equation by stochastic perturbation, Invent. Math., 180, 1-53, 2010.

[11] F. Flandoli, M. Gubinelli, E. Priola, Flow of diffeomorphisms for SDEs with unbounded Hölder continuous drift, Bulletin des Sciences Mathematiques, 134, 405-422, 2010.

[12] E. B. Fabes, B. F. Jones, N. M. Riviere, The initial value problem for the Navier-Stokes equations with data in $L_{p}$, Archive for Rational Mechanics and Analysis, 45, 222-240, 1972.

[13] H.Fujita, T. Kato, On the Navier-Stokes initial value problem I, Arch. Rational Mech. Anal. 16, 269-315, 1964.

[14] H.Fujita, T. Kato, On the non-stationary Navier-Stokes system, , Rend. Sem. Mat. Univ. Padova, 32, 243-260, 1962.

[15] Y. Giga: Solutions for semilinear parabolic equations in $L_{p}$ and regularity of weak solutions of the Navier-Stokes system, J. Differential Equations 62, 186-212, 1986.

[16] G. Iyer, A stochastic Lagrangian formulation of the Navier-Stokes and related transportequations, Ph. D. Thesis, University of Chicago, 2006.

[17] I. Karatzas, S. Shreve, Brownian Motion and Stochastic Calculus., New York, Springer-Verlag, 1988.

[18] N.V. Krylov, M. Röckner, Strong solutions to stochastic equations with singular time dependent drift, Probab. Theory Relat. Fields, 131, 154196, 2005.

[19] P.G. Lemarie-Rieusset, Recent developments in the Navier-Stokes problem, Chapman Hall/CRC, 2002.

[20] H. Sohr, The Navier-Stokes equations. An elementary functional analytic approach, Birkhäuser Advanced Texts, Birkhäuser Verlag, Basel, 2001.

[21] F Rezakhanlou, Stochastically symplectic maps and their applications to the Navier-Stokes equation, Annales de l'Institut Henri Poincare (C) Non Linear, 33, 1-22, 2016. 
[22] Temam, R., Navier-Stokes equations and nonlinear functional analysis, Second edition. CBMS-NSF Regional Conference Series in Applied Mathematics, 66. Society for Industrial and Applied Mathematics (SIAM), Philadelphia, PA, 1995.

[23] A. Veretennikov, Strong solutions and explicit formulas for solutions of stochastic integral equations (Russian). Mat Sb (N.S.) 111, 434-452, 1980 .

[24] X. Zhang, Stochastic Lagrangian particle approach to fractal NavierStokes equations, Communications in Mathematical Physics, 311, 133155, 2012.

[25] X. Zhang, A stochastic representation for backward incompressible Navier-Stokes equations, Prob. Theory and Rela. Fields, 148, 305$332,2010$.

[26] A. K. Zvonkin , A transformation of the phase space of a diffusion process that will remove the drift (Russian). Mat Sb (N.S.) 93, 129149, 152, 1974. 